\title{
Possibilistic Fuzzy Net Present Value Model and Application
}

\author{
S. S. Appadoo \\ Department of Supply Chain Management, Asper School of Business, Faculty of Management, University of Manitoba, \\ Winnipeg, MB, Canada R3T 5V4 \\ Correspondence should be addressed to S. S. Appadoo; ss.appadoo@ad.umanitoba.ca
}

Received 28 February 2014; Accepted 21 May 2014; Published 4 August 2014

Academic Editor: Pankaj Gupta

Copyright (c) 2014 S. S. Appadoo. This is an open access article distributed under the Creative Commons Attribution License, which permits unrestricted use, distribution, and reproduction in any medium, provided the original work is properly cited.

The cash flow values and the interest rate in the net present value (NPV) model are usually specified by either crisp numbers or random variables. In this paper, we first discuss some of the recent developments in possibility theory and find closed form expressions for fuzzy possibilistic net present value (FNPV). Then, following Carlsson and Fullér (2001), we discuss some of the possibilistic moments related to FNPV model along with an illustrative numerical example. We also give a unified approach to find higher order moments of FNPV by using the moment generating function introduced by Paseka et al. (2011).

\section{Introduction}

Recently, there has been growing interest in using fuzzy systems to deal with impreciseness, uncertainty, and vagueness (e.g., see Buckley [1], Kaufmann and Gupta [2], Zimmermann [3] etc.). Viewing the fuzzy numbers as random sets, Dubois and Prade [4], defined their interval valued expectation and introduced their mean value as a closed interval bounded by the expectations calculated from its upper and lower distribution functions. In recent years fuzzy systems has become an extensively area of research mainly due to the fact that deterministic models have huge limitations. Also, it has been shown that the decision making models based on probability theory are relatively hard to deal with due to their complex stochastic structure.

Possibility theory (Carlsson and Fullér [5]) along with fuzzy set theory and fuzzy systems (see (Zadeh [6]; Zimmermann [3]), Kaufmann and Gupta [2] provide a new avenue to deal with impreciseness in decision making problems. Recently, there have been several applications of possibility theory in decision making (e.g., see Appadoo et al. [7] and Thavaneswaran et al. [8]). Appadoo et al. [7], using possibilistic moments of adaptive fuzzy numbers, develop a model for fuzzy net present value (FNPV) of future cash flows. Paseka et al. [9] define moment generating function of fuzzy numbers and apply it to some time series models in finance, and Thavaneswaran et al. [8] introduce noncentered possibilistic moments, extend the results to centered moments, and find the kurtosis for a class of fuzzy coefficient autoregressive (FCA) and fuzzy coefficient volatility (FCV) models. Furthermore, they demonstrate the superiority of fuzzy forecasts over the least square error forecast. In this paper we revisit the fuzzy net value problem (FNVP) addressed extensively in the literature. However, these studies have, generally, been confined to fuzzy numbers having linear type of membership functions. The main reason for using linear membership function is to avoid complex nonlinear computations (for more details, e.g., see Medaglia et al. [10] and Medasani et al. [11]). Furthermore, they have pointed out that there are difficulties associated with the selection of the solution of a problem that uses linear membership function. They also have highlighted the importance of having a membership function which can be easily tuned and adjusted. In the present paper we use special type of fuzzy numbers, called $(m, n)$-trapezoidal fuzzy numbers [12], that have special type of nonlinear membership functions. Although the classical NPV method plays a decisive role in evaluation, it does not take into account the uncertainties which may be inherent in these parameters used in it. Ward [13] develops a fuzzy net present model by introducing trapezoidal fuzzy numbers as the future cash flow amounts. Due to computational efficiency Chiu and Park [14] modify the proposed fuzzy net present value formula by using triangular fuzzy numbers (TFNs) instead. Buckley [15] proposes fuzzy capital budgeting 
model in mathematics of finance. Kaufmann and Gupta [2] apply fuzzy discount rate to the fuzzy net present value model. Karsak and Tolga [16] present a fuzzy present value model for financial evaluation of advanced manufacturing system (AMS) investments under conditions of inflation. Kuchta [17] considers a global fuzzy net present value and uses quadratic 0-1 programming to the proposed model. Kahraman et al. [18] consider fuzzy present value, fuzzy equivalent uniform annual value, fuzzy final value, fuzzy benefit-cost ratio, and fuzzy payback period in the capital budgeting model. Liou and Chen [19] proposed a fuzzy equivalent uniform annual worth method to assist practitioners in evaluating investment alternatives. Omitaomu and Badiru [20] developed a fuzzy model for evaluating information system projects. Different from using possibility as the measure of a fuzzy event, recently Huang [21] and Huang [22] extended chance-constrained programming idea to fuzzy environment to solve fuzzy capital budgeting problems based on credibility measure. Furthermore, based on credibility theory, Huang [23] proposed models for selecting projects that combine random uncertainty and fuzzy uncertainty simultaneously.

In what follows, we divide the paper into 4 sections. In the first section we provide preliminaries, notation, and definitions. Main results are derived in Sections 2 and 3. In Section 2, we provide some moment properties of fuzzy numbers with special reference to a more generalized type of fuzzy numbers. Furthermore, in this section we consider possibilistic moment generating functions associated with fuzzy numbers. In Section 3 we consider fuzzy net present value problem (FNPV), state two easy to prove main results along with few special but useful cases, including the moment generating function associated with FNPV. In Section 4, we conclude the paper.

1.1. Preliminaries and Notation. In the sequel, we shall denote a classical set of objects, called the universe, by $X$ whose generic elements shall be denoted by $x$. A set of real numbers will be denoted by $\mathfrak{R}$, positive real numbers will be denoted by $\mathfrak{R}^{+}$, a fuzzy number will be denoted by by $A$, and a set of fuzzy numbers will be denoted by $\mathfrak{F}$. We now have the following definitions.

Definition 1. Fuzzy set $A$ in $X \subset \mathfrak{R}$, the set of real numbers, is a set of ordered pairs $A=\{x, \mu(x): x \in X\}$, where $\mu(x)$ is the membership function or grade of membership or degree of compatibility or degree of truth of $x \in X$ which maps $x \in X$ on the real interval $[0,1]$.

Definition 2. If $\operatorname{Sup} \mu(x)=1, x \in \mathfrak{R}$, then the fuzzy set $A$ is called a normal fuzzy set in $\Re$.

Definition 3. The crisp set of elements that belong to the fuzzy set $A$ at least to the degree $\alpha$ is called the $\alpha$-level set (or $\alpha$ cut); that is, $A(\alpha)=\left\{x \in X \mid \mu(x) \geq \alpha, \alpha \in \mathfrak{R}^{+}\right\}$. If the set $A^{\prime}(\alpha)=\left\{x \in X \mid \mu(x)>\alpha, \alpha \in \mathfrak{R}^{+}\right\}$, then $A^{\prime}(\alpha)$ is called strong $\alpha$-level set (or strong $\alpha$-cut).

Definition 4. A fuzzy set $A$ is said to be a convex set if $\mu\left(\lambda x_{1}+\right.$ $\left.(1-\lambda) x_{2}\right) \geq \min \left(\mu\left(x_{1}\right), \mu\left(x_{2}\right)\right), x_{1}, x_{2} \in X$ and $\lambda \in[0,1]$.
Definition 5. A fuzzy set $A$, which is both convex and normal, is defined to be a fuzzy number on the universal set $\mathfrak{R}$.

Definition 6. Let $A$ be a subset of $\mathfrak{R}$, then the set of elements having the largest degree of membership in $A$ is called the core of $A$. Thus,

$$
\operatorname{Core}(A)=\left\{x \in X \mid \mu_{A}(x)=\sup _{x \in X} \mu_{A}(x)\right\} .
$$

If $A$ is a fuzzy number $\in \mathfrak{F}$, where $\mathfrak{F}$ is the class of all fuzzy numbers then, by the definition of normality, $\operatorname{Core}(A) \neq 0$. Furthermore, $\operatorname{Core}(A)$ is also called the 1-level set of $A$. The support of a fuzzy set $A$ is a set of elements in $X$ for which $\mu_{A}(x)$ is positive; that is,

$$
\operatorname{Supp}(A)=\left\{x \in X \mid \mu_{A}(x)>0\right\} .
$$

Definition 7. An $\alpha$-level set of a fuzzy number $A$ is denoted by $A(\alpha)=\{t \in R \mid A(t) \geq \alpha\}$ if $\alpha>0$, and $A(\alpha)=c l\{t \in R \mid$ $A(t)>\alpha\}$, if $\alpha=0$.

Definition 8 (see Appadoo [12]). A fuzzy number $A=\left[a_{1}, a_{2}\right.$, $\left.a_{3}, a_{4}\right]_{(m, n)}, a_{1}<a_{2}<a_{3}<a_{4} \in X$, is said to be $(m, n)$ trapezoidal fuzzy number (written as $(m, n)$-Tr.F.N.) if its membership function $\mu(x)$ is given by

$$
\mu(x)= \begin{cases}0 & x \leq a_{1}, \\ 1-\left(\frac{a_{2}-x}{a_{2}-a_{1}}\right)^{m} & a_{1} \leq x \leq a_{2}, \\ 1 & a_{2} \leq x \leq a_{3}, \\ 1-\left(\frac{a_{3}-x}{a_{3}-a_{4}}\right)^{n} & a_{3} \leq x \leq a_{4}, \\ 0 & x \geq a_{4} .\end{cases}
$$

Setting $1-\left(\left(a_{2}-x\right) /\left(a_{2}-a_{1}\right)\right)^{m}=\alpha$ and $1-\left(\left(a_{3}-x\right) /\right.$ $\left.\left(a_{3}-a_{4}\right)\right)^{n}=\alpha$ we obtain the $\alpha$-cut representation of an $(m, n)$-Tr.F.N. $A=\left[a_{1}, a_{2}, a_{3}, a_{4}\right]_{(m, n)}$ as

$$
\begin{aligned}
& A(\alpha)=\left[a_{1}(\alpha), a_{2}(\alpha)\right] \\
&=\left[a_{2}-\left(a_{2}-a_{1}\right)(1-\alpha)^{1 / m}, a_{3}-\left(a_{3}-a_{4}\right)(1-\alpha)^{1 / n}\right], \\
& \forall \alpha \in(0,1] .
\end{aligned}
$$

Remark 9. It may be remarked here that as a special case, the results for a

(i) Tr.F.N. can be obtained from the results of an ( $m$, $n$ )-Tr.F.N., by setting $m=1$ and $n=1$, and

(ii) T.F.N. can be obtained from the results of an $(m$, $n$ )-Tr.F.N., by setting $m=1$ and $n=1$, along with $a_{3}=a_{2}$ and then writing $a_{3}$ for $a_{4}$.

Definition 10. An $(m, n)$-Tr.F.N. is said to be symmetric if $a_{2}-$ $a_{1}=a_{4}-a_{3}=\beta$ and $m=n$ in Definition 8 .

Remark 11. In view of Remark 9, the results for a symmetric Tr.F.N. and for a symmetric T.F.N. can be obtained from the results of a symmetric $(m, n)$-Tr.F.N. 


\section{Moment Properties of Fuzzy Numbers}

Following Carlsson and Fullér [5] we use the following results given in (5) in deriving the moment properties of $(m$, $n)$-Tr.F.N. Let $A \in \mathfrak{F}$ be a fuzzy number with $A(\alpha)=$ $\left[a_{1}(\alpha), a_{2}(\alpha)\right], \alpha \in[0,1]$. Following Carlsson and Fullér [5] and using properties (5) we have the following moment properties:

$$
\begin{aligned}
\operatorname{Possibility}\left[A \leq a_{1}(\alpha)\right] & =\pi\left(-\infty, a_{1}(\alpha)\right] \\
& =\sup _{u \leq a_{1}(\alpha)} A(u)=\alpha, \\
\text { Possibility }\left[A \geq a_{2}(\alpha)\right] & =\pi\left[a_{2}(\alpha), \infty\right) \\
& =\sup _{u \geq a_{2}(\alpha)} A(u)=\alpha .
\end{aligned}
$$

The lower possibilistic mean value $E_{L}(A)$, the upper possibilistic mean value $E_{R}(A)$, the possibilistic mean value $E(A)$, the interval value possibilistic mean $\operatorname{IVPM}(A)$, and the possibilistic variance $\operatorname{Var}(A)$ are below

$$
\begin{gathered}
E_{L}(A)=\frac{\int_{0}^{1} \operatorname{Pos}\left[A \leq a_{1}(\alpha)\right] a_{1}(\alpha) d \alpha}{\int_{0}^{1} \operatorname{Pos}\left[A \leq a_{1}(\alpha)\right] d \alpha}=2 \int_{0}^{1} \alpha a_{1}(\alpha) d \alpha, \\
E_{R}(A)=\frac{\int_{0}^{1} \operatorname{Pos}\left[A \geq a_{2}(\alpha)\right] a_{2}(\alpha) d \alpha}{\int_{0}^{1} \operatorname{Pos}\left[A \geq a_{2}(\alpha)\right] d \alpha}=2 \int_{0}^{1} \alpha a_{2}(\alpha) d \alpha, \\
E(A)=\int_{0}^{1}\left(a_{1}(\alpha)+a_{2}(\alpha)\right) \alpha d \alpha=\frac{E_{L}(A)+E_{R}(A)}{2},
\end{gathered}
$$

$$
\begin{aligned}
\operatorname{IVPM}(A) & =\left[E_{L}(A), E_{R}(A)\right] \\
& =\left[2 \int_{0}^{1} a_{1}(\alpha) \alpha d \alpha, 2 \int_{0}^{1} a_{2}(\alpha) \alpha d \alpha\right] .
\end{aligned}
$$

The variance of a fuzzy number $A$ is the expected value of the squared deviations between the arithmetic mean and the endpoints of its level sets. One has

$$
\begin{aligned}
\operatorname{Var}(A)= & \int_{0}^{1} \operatorname{Pos}\left[A \leq a_{1}\right]\left(\left[\frac{a_{1}(\alpha)+a_{2}(\alpha)}{2}-a_{1}(\alpha)\right]^{2}\right) d \alpha \\
& +\int_{0}^{1} \operatorname{Pos}\left[A \geq a_{2}\right]\left(\left[\frac{a_{1}(\alpha)+a_{2}(\alpha)}{2}-a_{2}(\alpha)\right]^{2}\right) d \alpha \\
= & \int_{0}^{1} \frac{1}{2}\left(a_{2}(\alpha)-a_{1}(\alpha)\right)^{2} \alpha d \alpha .
\end{aligned}
$$

Let $A$ and $B \in \mathfrak{F}$ be fuzzy numbers with $A(\alpha)=\left[a_{1}(\alpha), a_{2}(\alpha)\right]$ and $B(\alpha)=\left[b_{1}(\alpha), b_{2}(\alpha)\right], \alpha \in[0,1]$. Goetschel Jr. and Voxman [24] introduced a method for ranking fuzzy numbers as

$$
\begin{aligned}
A \leq B & \Longleftrightarrow \int_{0}^{1}\left(a_{1}(\alpha)+a_{2}(\alpha)\right) \alpha d \alpha \\
& \leq \int_{0}^{1}\left(b_{1}(\alpha)+b_{2}(\alpha)\right) \alpha d \alpha .
\end{aligned}
$$

As pointed out by Goetschel Jr. and Voxman [24] the definition given in (9) for ordering fuzzy numbers was motivated by the desire to give less importance to the lower levels of fuzzy numbers. Zhang and Nie [25] introduced the concepts of lower possibilistic and upper possibilistic variances of fuzzy numbers. These concepts are consistent with the extension principle and with the well-known definition of variance in probability theory. The lower and upper possibilistic variances of fuzzy number $A$ with $A(\alpha)=\left[a_{1}(\alpha), a_{2}(\alpha)\right]$, $\alpha \in[0,1]$ are as follows.

Remark 12. The lower possibilistic variance of $A$ is defined as the lower possibility-weighted average of the squared deviations between the left-hand endpoint and the lower possibilistic mean of its level sets. The lower possibilistic variance of $A$ is defined as

$$
\begin{aligned}
\operatorname{Var}_{L}(A) & =\frac{\int_{0}^{1} \operatorname{Pos}\left[A \leq a_{1}(\alpha)\right]\left(E_{L}(A)-a_{1}(\alpha)\right)^{2} d \alpha}{\int_{0}^{1} \operatorname{Pos}\left[A \leq a_{1}(\alpha)\right] d \alpha} \\
& =2 \int_{0}^{1} \alpha\left(E_{L}(A)-a_{1}(\alpha)\right)^{2} d \alpha .
\end{aligned}
$$

Remark 13. The upper possibilistic variance of $A$ is defined as the upper possibility-weighted average of the squared deviations between the right-hand endpoint and the upper possibilistic mean of its level sets. Therefore, one has the following:

$$
\begin{aligned}
\operatorname{Var}_{R}(A) & =\frac{\int_{0}^{1} \operatorname{Pos}\left[A \geq a_{1}(\alpha)\right]\left(E_{R}(A)-a_{2}(\alpha)\right)^{2} d \alpha}{\int_{0}^{1} \operatorname{Pos}\left[A \geq a_{2}(\alpha)\right] d \alpha} \\
& =2 \int_{0}^{1} \alpha\left(E_{R}(A)-a_{2}(\alpha)\right)^{2} d \alpha .
\end{aligned}
$$

Remark 14. The possibilistic variance of fuzzy number $A$ is defined as

$$
\begin{aligned}
\operatorname{Var}(A) & =\frac{\operatorname{Var}_{L}(A)+\operatorname{Var}_{R}(A)}{2} \\
& =\int_{0}^{1} \alpha\left(E_{L}(A)-a_{1}(\alpha)\right)^{2}+\left(E_{R}(A)-a_{2}(\alpha)\right)^{2} d \alpha .
\end{aligned}
$$

Remark 15. The crisp interval possibilistic variance of fuzzy number $A$ is defined as

$$
\begin{aligned}
\operatorname{ITPV}(A)= & {\left[\operatorname{Var}_{L}(A), \operatorname{Var}_{R}(A)\right] } \\
= & {\left[2 \int_{0}^{1} \alpha\left(E_{L}(A)-a_{1}(\alpha)\right)^{2} d \alpha,\right.} \\
& \left.2 \int_{0}^{1} \alpha\left(E_{R}(A)-a_{2}(\alpha)\right)^{2} d \alpha\right] .
\end{aligned}
$$

In the next subsection, in line with Carlsson and Fullér [5], we discuss possibilistic mean and possibilistic variance of $(m, n)$-Tr.F.N. For any $A \in \mathfrak{F}$ we use the notation $A(\alpha)=$ $\left[a_{1}(\alpha), a_{2}(\alpha)\right]$ for $\alpha$-level sets of $A$. 
TABLE 1: $E_{L}(A), E_{R}(A)$, and $E(A)$ for different values of $m$ and $n$.

\begin{tabular}{|c|c|c|c|c|}
\hline$m$ & $n$ & $E_{L}(A)$ & $E_{R}(A)$ & $E(A)$ \\
\hline 0.5 & 0.5 & $\frac{5}{6} a_{2}+\frac{1}{6} a_{1}$ & $\frac{5}{6} a_{3}+\frac{1}{6} a_{4}$ & $\frac{5}{12} a_{2}+\frac{1}{12} a_{1}+\frac{5}{12} a_{3}+\frac{1}{12} a_{4}$ \\
\hline 1 & 10 & $\frac{2}{3} a_{2}+\frac{1}{3} a_{1}$ & $\frac{31}{231} a_{3}+\frac{200}{231} a_{4}$ & $\frac{1}{3} a_{2}+\frac{1}{6} a_{1}+\frac{31}{462} a_{3}+\frac{100}{231} a_{4}$ \\
\hline 5 & 2 & $\frac{8}{33} a_{2}+\frac{25}{33} a_{1}$ & $\frac{7}{15} a_{3}+\frac{8}{15} a_{4}$ & $\frac{4}{33} a_{2}+\frac{7}{30} a_{3}+\frac{4}{15} a_{4}+\frac{25}{66} a_{1}$ \\
\hline 10 & 0.5 & $\frac{31}{231} a_{2}+\frac{200}{231} a_{1}$ & $\frac{5}{6} a_{3}+\frac{1}{6} a_{4}$ & $\frac{31}{462} a_{2}+\frac{5}{12} a_{3}+\frac{1}{12} a_{4}+\frac{100}{231} a_{1}$ \\
\hline 25 & 3 & $\frac{38}{663} a_{2}+\frac{625}{663} a_{1}$ & $\frac{5}{14} a_{3}+\frac{9}{14} a_{4}$ & $\frac{19}{663} a_{2}+\frac{5}{28} a_{3}+\frac{9}{28} a_{4}+\frac{625}{1326} a_{1}$ \\
\hline
\end{tabular}

2.1. Moments of Fuzzy Numbers $A=\left[a_{1}, a_{2}, a_{3}, a_{4}\right]_{\mathrm{O}(m, n)}$. Using (7) and (8) for the $(m, n)$-Tr.F.N., the possibilistic expected value and possibilistic variance are as follows;

$$
\begin{aligned}
E_{L}(A) & =\frac{2 a_{1} m^{2}+3 m a_{2}+a_{2}}{(1+2 m)(m+1)}, \\
E_{R}(A) & =\frac{2 n^{2} a_{4}+3 n a_{3}+a_{3}}{(1+2 n)(1+n)}, \\
E(A) & =\frac{2 a_{1} m^{2}+3 m a_{2}+a_{2}}{2(1+2 m)(m+1)}+\frac{2 n^{2} a_{4}+3 n a_{3}+a_{3}}{2(1+2 n)(1+n)} .
\end{aligned}
$$

It is important to point out here that when $A$ is a Tr.F.N. or a T.F.N., $E_{L}(A), E_{R}(A)$ and $E(A)$ can be easily obtained from (14). Similarly, using (10) and (11)

$\operatorname{Var}_{L}(A)$

$$
\begin{aligned}
= & 2 \int_{0}^{1} \alpha\left(E_{L}(A)-a_{1}(\alpha)\right)^{2} d \alpha=2 \int_{0}^{1} \alpha a_{1}^{2}(\alpha) d \alpha-E_{L}^{2}(A) \\
= & \left(\left(2 a_{2}^{2}+2 m^{3} a_{1}^{2}-2 m^{3} a_{2}^{2}-7 a_{2}^{2} m^{2}+a_{1}^{2} m^{2}+6 a_{2} a_{1} m^{2}\right.\right. \\
& \left.\left.+7 a_{2}^{2} m^{2}+7 a_{2}^{2} m+2 a_{2}^{2} m^{3}\right)\left(\left(3 m+2 m^{2}+1\right)(m+2)\right)^{-1}\right) \\
& -\left(\frac{2 a_{1} m^{2}+3 m a_{2}+a_{2}}{(1+2 m)(m+1)}\right)^{2},
\end{aligned}
$$

$\operatorname{Var}_{R}(A)$

$$
\begin{aligned}
= & 2 \int_{0}^{1} \alpha\left(E_{R}(A)-a_{1}(\alpha)\right)^{2} d \alpha=2 \int_{0}^{1} \alpha a_{2}^{2}(\alpha) d \alpha-E_{R}^{2}(A) \\
= & \left(\left(2 a_{3}^{2}-2 n^{3} a_{3}^{2}-7 n^{2} a_{3}^{2}+2 n^{3} a_{4}^{2}+a_{4}^{2} n^{2}+7 a_{3}^{2} n^{2}\right.\right. \\
& \left.\left.+2 a_{3}^{2} n^{3}+7 a_{3}^{2} n+6 n^{2} a_{4} a_{3}\right)((n+2)(1+n)(1+2 n))^{-1}\right) \\
& -\left(\frac{2 n^{2} a_{4}+3 n a_{3}+a_{3}}{(1+2 n)(1+n)}\right)^{2} .
\end{aligned}
$$

This yields

$$
\begin{aligned}
\operatorname{Var}(A)=( & \left(2 a_{2}^{2}+2 m^{3} a_{1}^{2}-2 m^{3} a_{2}^{2}-7 a_{2}^{2} m^{2}+a_{1}^{2} m^{2}\right. \\
& \left.+6 a_{2} a_{1} m^{2}+7 a_{2}^{2} m^{2}+7 a_{2}^{2} m+2 a_{2}^{2} m^{3}\right) \\
& \left.\times\left(2\left(3 m+2 m^{2}+1\right)(m+2)\right)^{-1}\right) \\
+ & \left(\left(2 a_{3}^{2}-2 n^{3} a_{3}^{2}-7 n^{2} a_{3}^{2}+2 n^{3} a_{4}^{2}+a_{4}^{2} n^{2}+7 a_{3}^{2} n^{2}\right.\right. \\
& \left.+2 a_{3}^{2} n^{3}+7 a_{3}^{2} n+6 n^{2} a_{4} a_{3}\right) \\
& \left.\times(2(n+2)(1+n)(1+2 n))^{-1}\right) \\
-( & \left.\frac{2 n^{2} a_{4}+3 n a_{3}+a_{3}}{2(1+2 n)(1+n)}\right)^{2}-\left(\frac{2 a_{1} m^{2}+3 m a_{2}+a_{2}}{2(1+2 m)(m+1)}\right)^{2} .
\end{aligned}
$$

For various values of $m$ and $n$ we obtain Tables 1,2 , and 3 .

2.2. Possibilistic Moment Generating Function. From Buckley [1] and Georgescu [26] we have that for an increasing function $g(x)$ and a fuzzy number $A$ whose $\alpha$-level sets are $A(\alpha)=$ $\left[a_{1}(\alpha), a_{2}(\alpha)\right]$ then we have the following:

$$
\begin{aligned}
(g(A))_{\alpha} & =\left\{g(x) \mid x \in(A)_{\alpha}\right\} \\
& =\left\{g(x) \mid a_{1}(\alpha) \leq x \leq a_{2}(\alpha)\right\} \\
& =\left\{g\left(a_{1}(\alpha)\right), g\left(a_{2}(\alpha)\right)\right\} .
\end{aligned}
$$

On the other hand if $g(x)$ is a decreasing function then

$$
\begin{aligned}
(g(A))_{\alpha} & =\left\{g(x) \mid x \in(A)_{\alpha}\right\} \\
& =\left\{g(x) \mid a_{2}(\alpha) \leq x \leq a_{1}(\alpha)\right\} \\
& =\left\{g\left(a_{2}(\alpha)\right), g\left(a_{1}(\alpha)\right)\right\} .
\end{aligned}
$$

The weighted possibilistic moment generating function, if exists, is defined as

$$
\operatorname{MGF}_{A}(u)=\frac{1}{2} \int_{0}^{1} f(\alpha)\left(e^{u\left(a_{1}(\alpha)\right)}+e^{u\left(a_{2}(\alpha)\right)}\right) d \alpha,
$$

$$
0 \leq \alpha \leq 1
$$


TABLE 2: $\operatorname{Var}_{L}(A)$ and $\operatorname{Var}_{R}(A)$ for different values of $m$ and $n$.

\begin{tabular}{lccc}
\hline$m$ & $n$ & $\operatorname{Var}_{L}(A)$ & $\operatorname{Var}_{R}(A)$ \\
\hline 0.5 & 0.5 & $\frac{7}{180} a_{2}^{2}-\frac{7}{90} a_{2} a_{1}+\frac{7}{180} a_{1}^{2}$ & $\frac{7}{180} a_{3}^{2}-\frac{7}{90} a_{3} a_{4}+\frac{7}{180} a_{4}^{2}$ \\
1 & 10 & $\frac{1}{18} a_{2}^{2}+\frac{1}{18} a_{1}^{2}-\frac{1}{9} a_{2} a_{1}$ & $\frac{425}{53361} a_{3}^{2}-\frac{850}{53361} a_{3} a_{4}+\frac{425}{53361} a_{4}^{2}$ \\
5 & 2 & $\frac{325}{15246} a_{2}^{2}-\frac{325}{7623} a_{2} a_{1}+\frac{325}{15246} a_{1}^{2}$ & $\frac{11}{225} a_{3}^{2}-\frac{22}{225} a_{3} a_{4}+\frac{11}{225} a_{4}^{2}$ \\
10 & 0.5 & $\frac{425}{53361} a_{2}^{2}-\frac{850}{53361} a_{2} a_{1}+\frac{425}{53361} a_{1}^{2}$ & $\frac{7}{180} a_{3}^{2}-\frac{7}{90} a_{3} a_{4}+\frac{7}{180} a_{4}^{2}$ \\
25 & 3 & $\frac{4375}{2637414} a_{2}^{2}-\frac{4375}{1318707} a_{2} a_{1}+\frac{4375}{2637414} a_{1}^{2}$ & $\frac{9}{245} a_{3}^{2}-\frac{18}{245} a_{3} a_{4}+\frac{9}{245} a_{4}^{2}$ \\
\hline
\end{tabular}

TABLE 3: $\operatorname{Var}(A)$ for different values of $m$ and $n$.

\begin{tabular}{ccc}
\hline$m$ & $n$ & $\operatorname{Var}(A)$ \\
\hline 0.5 & 0.5 & $\frac{7}{360} a_{2}^{2}-\frac{7}{180} a_{2} a_{1}+\frac{7}{360} a_{1}^{2}+\frac{7}{360} a_{3}^{2}-\frac{7}{180} a_{3} a_{4}+\frac{7}{360} a_{4}^{2}$ \\
1 & 10 & $\frac{1}{36} a_{2}^{2}+\frac{1}{36} a_{1}^{2}-\frac{1}{18} a_{2} a_{1}+\frac{425}{106722} a_{3}^{2}-\frac{425}{53361} a_{3} a_{4}+\frac{425}{106722} a_{4}^{2}$ \\
5 & 2 & $\frac{325}{30492} a_{2}^{2}-\frac{325}{15246} a_{2} a_{1}+\frac{325}{30492} a_{1}^{2}+\frac{11}{450} a_{3}^{2}-\frac{11}{225} a_{3} a_{4}+\frac{11}{450} a_{4}^{2}$ \\
10 & 0.5 & $\frac{425}{106722} a_{2}^{2}-\frac{425}{53361} a_{2} a_{1}+\frac{425}{106722} a_{1}^{2}+\frac{7}{360} a_{3}^{2}-\frac{7}{180} a_{3} a_{4}+\frac{7}{360} a_{4}^{2}$ \\
25 & 3 & $\frac{4375}{5274828} a_{2}^{2}-\frac{4375}{2637414} a_{2} a_{1}+\frac{4375}{5274828} a_{1}^{2}+\frac{9}{490} a_{3}^{2}-\frac{9}{245} a_{3} a_{4}+\frac{9}{490} a_{4}^{2}$ \\
\hline
\end{tabular}

As a special case if we assume that the weighting function is $f(\alpha)=2 \alpha$, then expression (19) can be rewritten in the following form:

$$
\begin{array}{r}
\operatorname{MGF}_{A}(u)=\int_{0}^{1} \alpha\left(e^{u\left(a_{1}(\alpha)\right)}+e^{u\left(a_{2}(\alpha)\right)}\right) d \alpha, \\
0 \leq \alpha \leq 1 .
\end{array}
$$

Based on (19), we define the weighted possibilistic moments as follows:

$$
E_{r}(A)=\left.\frac{d^{r}}{d u^{r}}\left(\operatorname{MGF}_{A}(u)\right)\right|_{u=0}=\lim _{u \rightarrow 0} \frac{d^{r}}{d t^{r}}\left(\operatorname{MGF}_{A}(u)\right) .
$$

In the following section, we formulate the possibilistic net present value model and discuss its possibilistic moment properties.

\section{Fuzzy Net Present Value}

Net present value (NPV) is a measure of economic effectiveness and is defined as the sum of the discounted net cash flows generated during consecutive years of the economic life of an investment opportunity. Consider the following:

$$
\begin{aligned}
\operatorname{NPV}(C, r, t)= & -C_{0}+\frac{C_{1}}{1+r_{1}}+\frac{C_{2}}{\left(1+r_{1}\right)\left(1+r_{2}\right)} \\
& +\cdots \frac{C_{n}}{\left(1+r_{1}\right)\left(1+r_{2}\right) \cdots\left(1+r_{n}\right)} \\
= & -C_{0}+\left(\sum_{t=1}^{n} \prod_{s=1}^{t} \frac{C_{t}}{\left(1+r_{s}\right)}\right) .
\end{aligned}
$$

$C_{0}$ is the net cash outflow at the beginning of a project which is a negative value, $C_{t}$ is the expected net cash inflow of the project estimated by the decision maker at $t$ th time period, and $r_{t}$ is the required rate of return or the discount rate of a project at $t$ th time period.

Remark 16. It is important to note here that if the required rate of return is constant for all $t>0$, then expression (22) reduces to

$$
\begin{aligned}
\operatorname{NPV}(C, r, t) & =-C_{0}+\frac{C_{1}}{(1+r)}+\frac{C_{2}}{(1+r)^{2}}+\cdots \frac{C_{n}}{(1+r)^{n}} \\
& =-C_{0}+\sum_{t=1}^{n} \frac{C_{t}}{(1+r)^{t}} .
\end{aligned}
$$


Below we give a theorem for the fuzzy net present value (see Appadoo et al. ([7, 27]) for details), where all the parameters, $C_{0}, \ldots, C_{n}$, and $r$, in the models are assumed to be fuzzy numbers and the corresponding $\alpha$-cuts for the FNPV model considered in Theorem 17 are given below. The following theorem extends the results in Appadoo et al. [7] to the time varying interest.

Theorem 17. For the fuzzy investment made at time period $t$ are given by $F N P V(C, r, t, \alpha)$; let

(a) $C_{t}(\alpha)=\left[C_{t 1}(\alpha), C_{t 2}(\alpha)\right], 0 \leq \alpha \leq 1, t=0,1,2, \ldots, n$ be the fuzzy investment made at the end of period $t$, and let

(b) $r_{t}(\alpha)=\left[r_{t 1}(\alpha), r_{t 2}(\alpha)\right], 0 \leq \alpha \leq 1, t=0,1,2, \ldots, n$ be the fuzzy return on the investment at each period $t$.

The cumulative discounted fuzzy net present value (FNPV) is given by

$$
\begin{array}{rl}
F N & P V(C, r, t, \alpha) \\
= & \left(-C_{02}(\alpha),-C_{01}(\alpha)\right)+\left(\frac{C_{11}(\alpha)}{1+r_{12}(\alpha)}, \frac{C_{12}(\alpha)}{1+r_{11}(\alpha)}\right) \\
& +\left(\frac{C_{21}(\alpha)}{\left(1+r_{12}(\alpha)\right)\left(1+r_{22}(\alpha)\right)},\right. \\
& \left.\frac{C_{22}(\alpha)}{\left(1+r_{11}(\alpha)\right)\left(1+r_{21}(\alpha)\right)}\right) \\
+ & +\left(\frac{C_{n 1}(\alpha)}{\left(1+r_{12}(\alpha)\right)\left(1+r_{22}(\alpha)\right) \cdots\left(1+r_{n 2}(\alpha)\right)},\right. \\
= & \left(-C_{02}(\alpha),-C_{01}(\alpha)\right) \\
& +\left(\sum_{t=1}^{n} \prod_{s=1}^{t} \frac{C_{t 1}(\alpha)}{\left(1+r_{s 2}(\alpha)\right)}, \sum_{t=1}^{n} \prod_{s=1}^{t} \frac{C_{t 2}(\alpha)}{\left(1+r_{s 1}(\alpha)\right)}\right) .
\end{array}
$$

Hence, the $\alpha$-cuts of lower and upper $F N P V(C, r, t, \alpha)$ are given by

$$
\begin{aligned}
& F N P V_{L}(C, r, t, \alpha)=-C_{02}(\alpha)+\sum_{t=1}^{n} \prod_{s=1}^{t} \frac{C_{t 1}(\alpha)}{\left(1+r_{s 2}(\alpha)\right)}, \\
& F N P V_{R}(C, r, t, \alpha)=-C_{01}(\alpha)+\sum_{t=1}^{n} \prod_{s=1}^{t} \frac{C_{t 2}(\alpha)}{\left(1+r_{s 1}(\alpha)\right)} .
\end{aligned}
$$

Corollary 18. In (24) if all the $C_{t}$ 's are crisps, for all t then one has

$F N P V(C, r, t, \alpha)$

$$
=\left(-C_{0},-C_{0}\right)+\left(\sum_{t=1}^{n} \prod_{s=1}^{t} \frac{C_{t}}{\left(1+r_{s 2}(\alpha)\right)}, \sum_{t=1}^{n} \prod_{s=1}^{t} \frac{C_{t}}{\left(1+r_{s 1}(\alpha)\right)}\right) .
$$

Corollary 19. In (24) if all the $r_{t}$ 's are crisps, for all then one has

$$
\begin{aligned}
\operatorname{FNPV}(C, r, t, \alpha)= & \left(-C_{02}(\alpha),-C_{01}(\alpha)\right) \\
& +\left(\sum_{t=1}^{n} \prod_{s=1}^{t} \frac{C_{t 1}(\alpha)}{\left(1+r_{s}\right)}, \sum_{t=1}^{n} \prod_{s=1}^{t} \frac{C_{t 2}(\alpha)}{\left(1+r_{s}\right)}\right) .
\end{aligned}
$$

Corollary 20. In (24) if all the $C_{t}$ 's are crisps and $r_{t}(\alpha)=$ $\left[r_{1}(\alpha), r_{2}(\alpha)\right]$, for all then one has

$$
\begin{array}{rl}
F & P V(C, r, t, \alpha) \\
& =\left(-C_{0},-C_{0}\right)+\left(\sum_{t=1}^{n} \frac{C_{t}}{\left(1+r_{2}(\alpha)\right)^{t}}, \sum_{t=1}^{n} \frac{C_{t}}{\left(1+r_{1}(\alpha)\right)^{t}}\right) .
\end{array}
$$

Below one discusses some possibilistic moment properties of FNPV as in (25) as follows.

Corollary 21. The lower possibilistic mean for FNPV is

$$
\begin{aligned}
E_{L} & (F N P V) \\
& =2 \int_{0}^{1} \alpha F N P V_{L}(C, r, t, \alpha) d \alpha \\
& =2 \int_{0}^{1} \alpha\left(-C_{02}(\alpha)+\sum_{t=1}^{n} \prod_{s=1}^{t} \frac{C_{t 1}(\alpha)}{\left(1+r_{s 2}(\alpha)\right)}\right) d \alpha .
\end{aligned}
$$

Corollary 22. The upper possibilistic mean for FNPV is

$$
\begin{aligned}
E_{R} & (F N P V) \\
& =2 \int_{0}^{1} \alpha F N P V_{R}(C, r, t, \alpha) d \alpha \\
& =2 \int_{0}^{1} \alpha\left(-C_{01}(\alpha)+\sum_{t=1}^{n} \prod_{s=1}^{t} \frac{C_{t 2}(\alpha)}{\left(1+r_{s 1}(\alpha)\right)}\right) d \alpha .
\end{aligned}
$$

Corollary 23. The interval value possibilistic mean for FNPV is

\section{$I V P M(F N P V)$}

$$
\begin{aligned}
= & {\left[E_{L}(F N P V), E_{R}(F N P V)\right] } \\
= & {\left[2 \int_{0}^{1} \alpha\left(-C_{02}(\alpha)+\sum_{t=1}^{n} \prod_{s=1}^{t} \frac{C_{t 1}(\alpha)}{\left(1+r_{s 2}(\alpha)\right)}\right) d \alpha,\right.} \\
& \left.2 \int_{0}^{1} \alpha\left(-C_{01}(\alpha)+\sum_{t=1}^{n} \prod_{s=1}^{t} \frac{C_{t 2}(\alpha)}{\left(1+r_{s 1}(\alpha)\right)}\right) d \alpha\right] .
\end{aligned}
$$

Corollary 24. The possibilistic mean for FNPV is

E (FNPV)

$$
\begin{gathered}
=\int_{0}^{1} \alpha\left(-C_{02}(\alpha)-C_{01}(\alpha)+\sum_{t=1}^{n} \prod_{s=1}^{t} \frac{C_{t 1}(\alpha)}{\left(1+r_{s 2}(\alpha)\right)}\right. \\
\left.+\sum_{t=1}^{n} \prod_{s=1}^{t} \frac{C_{t 2}(\alpha)}{\left(1+r_{s 1}(\alpha)\right)}\right) d \alpha .
\end{gathered}
$$


Corollary 25. Expression for lower possibilistic variance $\operatorname{Var}_{L}(F N P V)$ for FNPV is as follows:

$$
\begin{aligned}
& =\left(\int_{0}^{1} \operatorname{Pos}\left[F N P V \leq-C_{02}(\alpha)+\sum_{t=1}^{n} \prod_{s=1}^{t} \frac{C_{t 1}(\alpha)}{\left(1+r_{s 2}(\alpha)\right)}\right]\right. \\
& \times\left(E_{L}(F N P V)\right. \\
& \left.\quad-\left(-C_{02}(\alpha)+\sum_{t=1}^{n} \prod_{s=1}^{t} \frac{C_{t 1}(\alpha)}{\left(1+r_{s 2}(\alpha)\right)}\right)^{2} d \alpha\right) \\
& \times\left(\int_{0}^{1} \operatorname{Pos}\left[F N P V \leq-C_{02}(\alpha)+\sum_{t=1}^{n} \prod_{s=1}^{t} \frac{C_{t 1}(\alpha)}{\left(1+r_{s 2}(\alpha)\right)}\right] d \alpha\right)^{-1} \\
& =2 \int_{0}^{1} \alpha\left(E_{L}(F N P V)\right. \\
& \quad-\left(-C_{02}(\alpha)+\sum_{t=1}^{n} \prod_{s=1}^{t} \frac{C_{t 1}(\alpha)}{\left(1+r_{s 2}(\alpha)\right)}\right)^{2} d \alpha .
\end{aligned}
$$

Corollary 26. Similarly, one can give expression for upper possibilistic variance $\operatorname{Var}_{R}(F N P V)$ for FNPV is as follows:

$$
\begin{aligned}
& =\left(\int_{0}^{1} \operatorname{Pos}\left[F N P V \geq-C_{01}(\alpha)+\sum_{t=1}^{n} \prod_{s=1}^{t} \frac{C_{t 2}(\alpha)}{\left(1+r_{s 1}(\alpha)\right)}\right]\right. \\
& \times\left(E_{R}(F N P V)\right. \\
& \left.\left.\quad-\left(-C_{01}(\alpha)+\sum_{t=1}^{n} \prod_{s=1}^{t} \frac{C_{t 2}(\alpha)}{\left(1+r_{s 1}(\alpha)\right)}\right)\right)^{2} d \alpha\right) \\
& \times\left(\int _ { 0 } ^ { 1 } P ^ { 2 } \left[F N P V \geq-C_{01}(\alpha)\right.\right. \\
& \left.\left.\left.\quad-\left(-\sum_{t=1}^{n} \prod_{s=1}^{t} \frac{C_{t 2}(\alpha)}{\left(1+r_{s 1}(\alpha)\right)}\right] d \alpha\right)^{-1}(\alpha) \sum_{t=1}^{n} \prod_{s=1}^{t} \frac{C_{t 2}(\alpha)}{\left(1+r_{s 1}(\alpha)\right)}\right)\right)^{2} d \alpha \\
& =2 \int_{0}^{1} \alpha\left(E_{R}(F N P V)\right.
\end{aligned}
$$

Corollary 27. The interval value possibilistic variance $\operatorname{IVPV}(F N P V)$ is

$$
\begin{aligned}
& I V P V(F N P V) \\
& =\left[\operatorname{Var}_{L}(F N P V), \operatorname{Var}_{R}(F N P V)\right] \\
& =\left[2 \int _ { 0 } ^ { 1 } \alpha \left(E_{L}(F N P V)\right.\right. \\
& \left.\quad-\left(-C_{02}(\alpha)+\sum_{t=1}^{n} \prod_{s=1}^{t} \frac{C_{t 1}(\alpha)}{\left(1+r_{s 2}(\alpha)\right)}\right)\right)^{2} d \alpha \\
& \left.\left.\quad-\left(-C_{01}^{1}(\alpha)+\sum_{t=1}^{n} \prod_{s=1}^{t} \frac{C_{t 2}(\alpha)}{\left(1+r_{s 1}(\alpha)\right)}\right)\right)^{2} d \alpha\right] .
\end{aligned}
$$

Corollary 28. Thus, the possibilistic variance is

$$
\begin{aligned}
\operatorname{Var}(F N P V) & \\
=\int_{0}^{1} \alpha( & \left(E_{L}(F N P V)\right. \\
& \left.-\left(-C_{02}(\alpha)+\sum_{t=1}^{n} \prod_{s=1}^{t} \frac{C_{t 1}(\alpha)}{\left(1+r_{s 2}(\alpha)\right)}\right)\right)^{2} \\
& +\left(E_{R}(F N P V)\right. \\
& \left.\left.-\left(-C_{01}(\alpha)+\sum_{t=1}^{n} \prod_{s=1}^{t} \frac{C_{t 2}(\alpha)}{\left(1+r_{s 1}(\alpha)\right)}\right)\right)^{2}\right) d \alpha
\end{aligned}
$$

The possibilistic moment generating function for FNPV is as follows.

Corollary 29. Consider

$$
\begin{aligned}
& \operatorname{MGF}_{F N P V}(u) \\
& =\int_{0}^{1} \alpha\left(e^{u\left(F N P V_{L}(C, r, t, \alpha)\right)}+e^{u\left(F N P V_{R}(C, r, t, \alpha)\right)}\right) d \alpha \\
& =\int_{0}^{1} \alpha\left(e^{u\left(-C_{02}(\alpha)+\sum_{t=1}^{n} \prod_{s=1}^{t}\left(C_{t 1}(\alpha) /\left(1+r_{s 2}(\alpha)\right)\right)\right)}\right. \\
& \left.\quad+e^{u\left(-C_{01}(\alpha)+\sum_{t=1}^{n} \prod_{s=1}^{t}\left(C_{t 2}(\alpha) /\left(1+r_{s 1}(\alpha)\right)\right)\right)}\right) d \alpha,
\end{aligned}
$$


$E_{r}(F N P V)$

$$
\begin{array}{r}
=\left.\frac{d^{r}}{d t^{r}}\left(M G F_{F N P V}(u)\right)\right|_{t=0}=\lim _{u \rightarrow 0} \frac{d^{r}}{d t^{r}}\left(M G F_{F N P V}(u)\right) \\
=\lim _{u \rightarrow 0} \frac{d^{r}}{d t^{r}}\left(\int _ { 0 } ^ { 1 } \alpha \left(e^{u\left(-C_{02}(\alpha)+\sum_{t=1}^{n} \prod_{s=1}^{t}\left(C_{t 1}(\alpha) /\left(1+r_{s 2}(\alpha)\right)\right)\right)}\right.\right. \\
\left.\left.+e^{u\left(-C_{01}(\alpha)+\sum_{t=1}^{n} \prod_{s=1}^{t}\left(C_{t 2}(\alpha) /\left(1+r_{s 1}(\alpha)\right)\right)\right)}\right) d \alpha\right) .
\end{array}
$$

Below, one gives Theorem 30 that can be proved easily following Theorem 17.

\section{Theorem 30. Let}

(a) $C_{t}(\alpha)=\left[C_{t 1}(\alpha), C_{t 2}(\alpha)\right]=\left[C_{t 2}-\left(C_{t 2}-C_{t 1}\right)(1-\right.$ $\left.\alpha)^{1 / m_{t}}, C_{t 3}-\left(C_{t 3}-C_{t 4}\right)(1-\alpha)^{1 / n_{t}}\right], 0 \leq \alpha \leq 1$, $t=0,1,2, \ldots, n$ be the fuzzy investment made at the end of period $t$ and let

(b) $r_{t}(\alpha)=\left[r_{t 1}(\alpha), r_{t 2}(\alpha)\right]=\left[r_{t 2}-\left(r_{t 2}-r_{t 1}\right)(1-\alpha)^{1 / M_{t}}, r_{t 3}-\right.$ $\left.\left(r_{t 3}-r_{t 4}\right)(1-\alpha)^{1 / N_{t}}\right], 0 \leq \alpha \leq 1, t=0,1,2, \ldots, n$ be the fuzzy return on the investment at each of period $t$.

Then, the cumulative discounted fuzzy net present value (FNPV) is given by

$F N P V(C, r, t, \alpha)$

$$
\begin{aligned}
= & \left(\left(C_{03}-C_{04}\right)(1-\alpha)^{1 / n_{0}}-C_{03},\right. \\
& \left.\left(C_{02}-C_{01}\right)(1-\alpha)^{1 / m_{0}}-C_{02}\right) \\
+ & \left(\sum_{t=1}^{n} \prod_{s=1}^{t} \frac{C_{t 2}-\left(C_{t 2}-C_{t 1}\right)(1-\alpha)^{1 / m_{t}}}{\left(1+r_{s 3}-\left(r_{s 3}-r_{s 4}\right)(1-\alpha)^{1 / N_{s}}\right)},\right. \\
& \left.\sum_{t=1}^{n} \prod_{s=1}^{t} \frac{C_{t 3}-\left(C_{t 3}-C_{t 4}\right)(1-\alpha)^{1 / n_{t}}}{\left(1+r_{s 2}-\left(r_{s 2}-r_{s 1}\right)(1-\alpha)^{1 / M_{s}}\right)}\right),
\end{aligned}
$$

where

$$
\begin{aligned}
F N P V_{L}(C, r, t, \alpha) & \\
= & \left(C_{03}-C_{04}\right)(1-\alpha)^{1 / n_{0}}-C_{03} \\
& +\sum_{t=1}^{n} \prod_{s=1}^{t} \frac{C_{t 2}-\left(C_{t 2}-C_{t 1}\right)(1-\alpha)^{1 / m_{t}}}{\left(1+r_{s 3}-\left(r_{s 3}-r_{s 4}\right)(1-\alpha)^{1 / N_{s}}\right)}, \\
F N P V_{R}(C, r, t, \alpha) & \\
= & \left(C_{02}-C_{01}\right)(1-\alpha)^{1 / m_{0}}-C_{02} \\
& +\sum_{t=1}^{n} \prod_{s=1}^{t} \frac{C_{t 3}-\left(C_{t 3}-C_{t 4}\right)(1-\alpha)^{1 / n_{t}}}{\left(1+r_{s 2}-\left(r_{s 2}-r_{s 1}\right)(1-\alpha)^{1 / M_{s}}\right)} .
\end{aligned}
$$

We may now point out that one can easily study the possibilistic of structure $\operatorname{FNPV}(C, r, t, \alpha)$ since we have close form expressions for $\operatorname{FNPV}_{L}(C, r, t, \alpha)$ and $\operatorname{FNPV}_{R}(C, r, t, \alpha)$, respectively. Thus, we observe that it can be used to determine whether an investment is risky or not and this will be the subject of future research.

Below, we discussed an application of $\operatorname{FNPV}_{L}(C, r, t, \alpha)$ and $\operatorname{FNPV}_{R}(C, r, t, \alpha)$. It is important to point out here that data in the example given below can usually be obtained through expert opinion or survey. This approach is consistent with empirical studies in social science and in real life. Note that input data's in fuzzy modeling can also be data driven and there are genetic algorithms available which offer convenient ways to model fuzzy systems and membership functions from raw data. In the example below, we are more interested to give an example to show how the various possibilistic moments can be computed once the various model parameters are obtained.

Example 31. In this example we illustrate the application of fuzzy sets theory, possibility theory, and $(m, n)$-trapezoidal fuzzy numbers to the fuzzy net present value model developed early in the paper. Suppose we have an investment opportunity where the cash flows are given as fuzzy numbers as in Table 4, the fuzzy discount rate is given is Table 5. Based on information given in Table 4 and Table 5 we can calculate the fuzzy net present value using (39). It is worth noting that the input variables in (40), which can only be realized in the future, are full of uncertainty. In an uncertain decision making environment, input parameters are vague, ambiguous, and imprecise, which can only be handled by the fuzzy analysis as proposed in this paper.

Consider the following:

$$
\begin{aligned}
\operatorname{FNPV}_{L} & (C, r, t, \alpha) \\
=( & \left.-1000-50(1-\alpha)^{1 / n_{0}}\right)+\left(\frac{500-100(1-\alpha)^{1 / m_{1}}}{1.15+0.1(1-\alpha)^{1 / N_{1}}}\right) \\
+ & \left(\frac{\left(350-50(1-\alpha)^{1 / m_{2}}\right)}{\left(1.15+0.1(1-\alpha)^{1 / N_{1}}\right)\left(1.07+0.02(1-\alpha)^{1 / N_{2}}\right)}\right) \\
+ & \left(\left(150-50(1-\alpha)^{1 / m_{3}}\right)\right. \\
& \times\left(\left(1.15+0.1(1-\alpha)^{1 / N_{1}}\right)\left(1.07+0.02(1-\alpha)^{1 / N_{2}}\right)\right. \\
& \left.\left.\quad \times\left(1.08+0.01(1-\alpha)^{1 / N_{3}}\right)\right)^{-1}\right) \\
+ & \left(\left(\left(250-50(1-\alpha)^{1 / m_{4}}\right)\right.\right. \\
& \times\left(\left(1.08+0.01(1-\alpha)^{1 / N_{3}}\right)\right. \\
& \left.\left.\quad \times\left(1.04+0.02(1-\alpha)^{1 / N_{4}}\right)\right)^{-1}\right) \\
& \times\left(\left(1.15+0.1(1-\alpha)^{1 / N_{1}}\right)\right. \\
& \left.\left.\quad \times\left(1.07+0.02(1-\alpha)^{1 / N_{2}}\right)\right)^{-1}\right)
\end{aligned}
$$


TABLE 4: Fuzzy cash flow and corresponding $\alpha$-cuts, $\forall, \alpha, 0 \leq \alpha \leq 1$.

\begin{tabular}{lcc}
\hline Year $(i)$ & Fuzzy cash flows & Corresponding $\alpha$-cuts \\
\hline 0 & {$[800,900,1000,1050]_{O\left(m_{0}, n_{0}\right)}$} & $\left(900-100(1-\alpha)^{1 / m_{0}}, 1000+50(1-\alpha)^{1 / n_{0}}\right)$ \\
1 & {$[400,500,600,650]_{O\left(m_{1}, n_{1}\right)}$} & $\left(500-100(1-\alpha)^{1 / m_{1}}, 600+50(1-\alpha)^{1 / n_{1}}\right)$ \\
2 & {$[300,350,400,450]_{O\left(m_{2}, n_{2}\right)}$} & $\left(350-50(1-\alpha)^{1 / m_{2}}, 400+50(1-\alpha)^{1 / n_{2}}\right)$ \\
3 & {$[100,150,200,250]_{O\left(m_{3}, n_{3}\right)}$} & $\left(150-50(1-\alpha)^{1 / m_{3}}, 200+50(1-\alpha)^{1 / n_{3}}\right)$ \\
4 & {$[200,250,300,350]_{O\left(m_{4}, n_{4}\right)}$} & $\left(250-50(1-\alpha)^{1 / m_{4}}, 300+50(1-\alpha)^{1 / n_{4}}\right)$ \\
\hline
\end{tabular}

TABLE 5: Fuzzy discount rates and corresponding $\alpha$-cuts, $\forall, \alpha, 0 \leq \alpha \leq 1$.

\begin{tabular}{lcc}
\hline Year $(i)$ & Fuzzy discount rates & Corresponding $\alpha$-cuts \\
\hline 1 & {$[1.0,1.1,1.15,1.25]_{\mathrm{O}\left(M_{1}, N_{1}\right)}$} & $\left(1.1-0.1(1-\alpha)^{1 / M_{1}}, 1.15+0.1(1-\alpha)^{1 / N_{1}}\right)$ \\
2 & {$[1.03,1.05,1.07,1.09]_{O\left(M_{2}, N_{2}\right)}$} & $\left(1.03-0.02(1-\alpha)^{1 / M_{1}}, 1.07+0.02(1-\alpha)^{1 / N_{2}}\right)$ \\
3 & {$[1.05,1.07,1.08,1.09]_{\mathrm{O}\left(M_{3}, N_{3}\right)}$} & $\left(1.05-0.02(1-\alpha)^{1 / M_{3}}, 1.08+0.01(1-\alpha)^{1 / N_{3}}\right)$ \\
4 & {$[1.02,1.03,1.04,1.06]_{O\left(M_{4}, N_{4}\right)}$} & $\left(1.02-0.01(1-\alpha)^{1 / M_{4}}, 1.04+0.02(1-\alpha)^{1 / N_{4}}\right)$ \\
\hline
\end{tabular}

TABLE 6: Fuzzy net present value $\alpha$-cuts, $\forall, \alpha, 0 \leq \alpha \leq 1$.

\begin{tabular}{|c|c|c|c|c|c|c|c|c|c|c|c|c|}
\hline$m_{0}$ & 1 & 1 & 1 & 1 & 0.02 & 0.02 & 0.00002 & 0.00002 & 0.00002 & 0.00002 & 1000 & 1000 \\
\hline$m_{1}$ & 1 & 1 & 2 & 2 & 0.05 & 0.05 & 1 & 1 & 0.00002 & 0.00002 & 1000 & 1000 \\
\hline$m_{2}$ & 1 & 1 & 1 & 1 & 0.2 & 0.2 & 25 & 25 & 0.00002 & 0.00002 & 1000 & 1000 \\
\hline$m_{3}$ & 1 & 1 & 5 & 5 & 0.3 & 0.3 & 1 & 1 & 0.00002 & 0.00002 & 1000 & 1000 \\
\hline$m_{4}$ & 1 & 1 & 1 & 1 & 0.8 & 0.8 & 1 & 1 & 0.00002 & 0.00002 & 1000 & 1000 \\
\hline$n_{0}$ & 1 & 1 & 1 & 1 & 0.003 & 0.003 & 1 & 1 & 0.00002 & 0.00002 & 1000 & 1000 \\
\hline$n_{1}$ & 1 & 1 & 12 & 12 & 0.002 & 0.002 & 1 & 1 & 0.00002 & 0.00002 & 1000 & 1000 \\
\hline$n_{2}$ & 1 & 1 & 1 & 1 & 0.05 & 0.05 & 1 & 1 & 0.00002 & 0.00002 & 1000 & 1000 \\
\hline$n_{3}$ & 1 & 1 & 18 & 18 & 0.6 & 0.6 & 1 & 1 & 0.00002 & 0.00002 & 1000 & 1000 \\
\hline$n_{4}$ & 1 & 1 & 1 & 1 & 0.08 & 0.08 & 1000 & 1000 & 0.00002 & 0.00002 & 1000 & 1000 \\
\hline$M_{0}$ & 1 & 1 & 2.5 & 2.5 & 0.06 & 0.06 & 1 & 1 & 0.00002 & 0.00002 & 1000 & 1000 \\
\hline$M_{1}$ & 1 & 1 & 1 & 1 & 0.09 & 0.09 & 1 & 1 & 0.00002 & 0.00002 & 1000 & 1000 \\
\hline$M_{2}$ & 1 & 1 & 3.2 & 3.2 & 0.05 & 0.05 & 0.0009 & 0.0009 & 0.00002 & 0.00002 & 1000 & 1000 \\
\hline$M_{3}$ & 1 & 1 & 1 & 1 & 0.02 & 0.02 & 1 & 1 & 0.00002 & 0.00002 & 1000 & 1000 \\
\hline$M_{4}$ & 1 & 1 & 9 & 9 & 0.005 & 0.005 & 68 & 68 & 0.00002 & 0.00002 & 1000 & 1000 \\
\hline$N_{1}$ & 1 & 1 & 1 & 1 & 0.09 & 0.09 & 45 & 45 & 0.00002 & 0.00002 & 1000 & 1000 \\
\hline$N_{2}$ & 1 & 1 & 12 & 12 & 0.05 & 0.05 & 1 & 1 & 0.00002 & 0.00002 & 1000 & 1000 \\
\hline$N_{3}$ & 1 & 1 & 1 & 1 & 0.003 & 0.003 & 1 & 1 & 0.00002 & 0.00002 & 1000 & 1000 \\
\hline$N_{4}$ & 1 & 1 & 8 & 8 & 0.01 & 0.01 & 1 & 1 & 0.00002 & 0.00002 & 1000 & 1000 \\
\hline$\alpha$ & $\mathrm{FNPV}_{1}$ & $\mathrm{FNPV}_{2}$ & $\mathrm{FNPV}_{1}$ & $\mathrm{FNPV}_{2}$ & $\mathrm{FNPV}_{1}$ & $\mathrm{FNPV}_{2}$ & $\mathrm{FNPV}_{1}$ & $\mathrm{FNPV}_{2}$ & FFNPV $_{1}$ & $\mathrm{FNPV}_{2}$ & $\mathrm{FNPV}_{1}$ & $\mathrm{FNPV}_{2}$ \\
\hline 0 & -315.44 & 868.97 & -315.44 & 868.97 & -315.44 & 868.97 & -315.44 & 868.97 & -315.44 & 868.97 & -315.44 & 868.97 \\
\hline 0.1 & -284.93 & 819.76 & -292.48 & 830.35 & -105.05 & 507.69 & -314.47 & 868.00 & 12.98 & 413.85 & -315.40 & 868.92 \\
\hline 0.2 & -253.94 & 771.43 & -268.90 & 792.21 & -53.60 & 456.38 & -313.39 & 866.92 & 12.98 & 413.85 & -315.37 & 868.86 \\
\hline 0.3 & -222.46 & 723.97 & -244.63 & 754.49 & -30.16 & 439.88 & -312.18 & 865.70 & 12.98 & 413.85 & -315.33 & 868.79 \\
\hline 0.4 & -190.46 & 677.35 & -219.54 & 717.15 & -16.44 & 432.27 & -310.78 & 864.30 & 12.98 & 413.85 & -315.28 & 868.72 \\
\hline 0.5 & -157.94 & 631.54 & -193.47 & 680.10 & -7.28 & 427.15 & -309.14 & 862.65 & 12.98 & 413.85 & -315.23 & 868.63 \\
\hline 0.6 & -124.89 & 586.51 & -166.20 & 643.25 & -0.73 & 422.98 & -307.14 & 860.65 & 12.98 & 413.85 & -315.16 & 868.52 \\
\hline 0.7 & -91.29 & 542.25 & -137.31 & 606.40 & 4.17 & 419.50 & -304.57 & 858.08 & 12.98 & 413.85 & -315.07 & 868.37 \\
\hline 0.8 & -57.12 & 498.74 & -105.99 & 569.17 & 7.95 & 416.72 & -301.00 & 854.50 & 12.98 & 413.85 & -314.95 & 868.17 \\
\hline 0.9 & -22.37 & 455.94 & -70.10 & 530.39 & 10.92 & 414.75 & -294.98 & 848.49 & 12.98 & 413.85 & -314.74 & 867.83 \\
\hline 1 & 12.98 & 413.85 & 12.98 & 413.85 & 12.98 & 413.85 & 12.98 & 413.85 & 12.98 & 413.85 & 12.98 & 413.85 \\
\hline
\end{tabular}


and similarly,

$$
\begin{aligned}
& \operatorname{FNPV}_{R}(C, r, t, \alpha) \\
& =\left(-900+100(1-\alpha)^{1 / m_{0}}\right)+\left(\frac{600+50(1-\alpha)^{1 / n_{1}}}{1.1-0.1(1-\alpha)^{1 / M_{1}}}\right) \\
& +\left(\frac{\left(400+50(1-\alpha)^{1 / n_{2}}\right)}{\left(1.1-0.1(1-\alpha)^{1 / M_{1}}\right)\left(1.03-0.02(1-\alpha)^{1 / M_{2}}\right)}\right) \\
& +\left(\left(200+50(1-\alpha)^{1 / n_{3}}\right)\right. \\
& \times\left(\left(1.1-0.1(1-\alpha)^{1 / M_{1}}\right)\left(1.03-0.02(1-\alpha)^{1 / M_{2}}\right)\right. \\
& \left.\left.\times\left(1.05-0.02(1-\alpha)^{1 / M_{3}}\right)\right)^{-1}\right) \\
& +\left(\left(\left(300+50(1-\alpha)^{1 / n_{4}}\right)\right.\right. \\
& \times\left(\left(1.05-0.02(1-\alpha)^{1 / M_{3}}\right)\right. \\
& \left.\left.\times\left(1.02-0.01(1-\alpha)^{1 / M_{4}}\right)\right)^{-1}\right) \\
& \times\left(\left(1.1-0.1(1-\alpha)^{1 / M_{1}}\right)\right. \\
& \left.\left.\times\left(1.03-0.02(1-\alpha)^{1 / M_{2}}\right)\right)^{-1}\right) ;
\end{aligned}
$$

we can easily compute $\operatorname{FNPV}_{L}(C, r, t, \alpha)$ and $\operatorname{FNPV}_{R}(C, r$, $t, \alpha$ ) for different values of $\alpha$ for $0 \leq \alpha \leq 1$. As a simple illustration it is easy to see that when $m_{0}=0.00002, m_{1}=1$, $m_{2}=25, m_{3}=1, m_{4}=1, n_{0}=1, n_{1}=1, n_{2}=1$, $n_{3}=1, n_{4}=1000, M_{1}=1, M_{2}=0.0009, M_{3}=1$, $M_{4}=68, N_{0}=45, N_{1}=1, N_{2}=1, N_{3}=1$, and $\alpha=0.7$ we have $\mathrm{FNPV}_{L}=-304.57$ and $\mathrm{FNPV}_{R}=858.08$ for detail see Table 6. As in Appadoo et al. [7] we can also compute possibilistic moments of $\operatorname{FNPV}_{L}(C, r, t, \alpha)$ and $\mathrm{FNPV}_{R}(C, r, t, \alpha)$. Most of the fuzzy net present value models have been confined to linear type of fuzzy numbers. As pointed out by Medaglia et al. [10] and Medasani et al. [11], even though these linear membership functions do provide ease in calculations, they do not represent precisely the linguistic terms being modeled. There are many difficulties associated with selecting the solution of a problem written in linear membership functions and they have highlighted the importance of using nonlinear membership functions that can be easily tuned and adjusted $([10,11])$. This example shows clearly that $\operatorname{FNPV}_{L}(C, r, t, \alpha)$ and $\operatorname{FNPV}_{R}(C, r, t, \alpha)$ depend on the curvature of the membership function. For different values of the curvatures we obtained different values of $\operatorname{FNPV}(C, r, t, \alpha)$.

\section{Conclusion}

The fuzzy net present value model developed in this paper is practical and useful. The methodology proposed in this paper may also be applicable to other fuzzy cash flow models as well. This model could provide investors with a better understanding of the cash flow model when making investment decisions. The evidence in favor of a fuzzy approach highlights the advantage over the original crisp version of the net present value model. Our approach provides a formulation that more closely conforms to real situation. The proposed fuzzy model does not imply rejection of other discounted cash formulation but rather compliments existing fuzzy model formulation.

\section{Conflict of Interests}

The author declares that there is no conflict of interests regarding the publication of this paper.

\section{References}

[1] J. J. Buckley, Fuzzy Probabilities, Studies in Fuzziness and Soft Computing, Springer, Berlin, Germany, 2005.

[2] A. Kaufmann and M. M. Gupta, Introduction to Fuzzy Arithmetic Theory and Applications, Von Nostrand Reinhold, New York, NY, USA, 1985.

[3] H.-J. Zimmermann, Fuzzy Sets Theory and Its Applications, Kluwer Academic Publishers, Nowell, Mass, USA, 4th edition, 2001.

[4] D. Dubois and H. Prade, "The mean value of a fuzzy number," Fuzzy Sets and Systems, vol. 24, no. 3, pp. 279-300, 1987.

[5] C. Carlsson and R. Fullér, "On possibilistic mean value and variance of fuzzy numbers," Fuzzy Sets and Systems, vol. 122, no. 2, pp. 315-326, 2001

[6] L. A. Zadeh, "Fuzzy sets," Information and Computation, vol. 8, pp. 338-353, 1965.

[7] S. S. Appadoo, S. K. Bhatt, and C. R. Bector, "Application of possibility theory to investment decisions," Fuzzy Optimization and Decision Making, vol. 7, no. 1, pp. 35-57, 2008.

[8] A. Thavaneswaran, S. S. Appadoo, and A. Paseka, "Weighted possibilistic moments of fuzzy numbers with applications to GARCH modeling and option pricing," Mathematical and Computer Modelling, vol. 49, no. 1-2, pp. 352-368, 2009.

[9] A. Paseka, S. S. Appadoo, and A. Thavaneswaran, "Possibilistic moment generating functions," Applied Mathematics Letters, vol. 24, no. 5, pp. 630-635, 2011.

[10] A. L. Medaglia, S.-C. Fang, H. L. W. Nuttle, and J. R. Wilson, "An efficient and flexible mechanism for constructing membership functions," European Journal of Operational Research, vol. 139, no. 1, pp. 84-95, 2002.

[11] S. Medasani, J. Kim, and R. Krishnapuram, "An overview of membership function generation techniques for pattern recognition," International Journal of Approximate Reasoning, vol. 19, no. 3-4, pp. 391-417, 1998.

[12] S. S. Appadoo, Pricing financial derivatives with fuzzy algebraic models: a theoretical and computational approach [Ph.D. thesis], University of Manitoba, Winnipeg, Manitoba, Canada, 2006.

[13] T. L. Ward, "Discounted fuzzy cash ow analysis," in Proceedings of the Fall Industrial Engineering Conference, pp. 476-481, Institute of Industrial Engineers, 1985.

[14] C.-Y. Chiu and C. S. Park, "Fuzzy cash flow analysis using present worth criterion," The Engineering Economist, vol. 39, no. 2, pp. 113-138, 1994.

[15] J. J. Buckley, "The fuzzy mathematics of finance," Fuzzy Sets and Systems, vol. 21, no. 3, pp. 257-273, 1987. 
[16] E. Ertugrul Karsak and E. Tolga, "Fuzzy multi-criteria decisionmaking procedure for evaluating advanced manufacturing system investments," International Journal of Production Economics, vol. 69, no. 1, pp. 49-64, 2001.

[17] D. Kuchta, "A fuzzy model for R\&D project selection with benefit, outcome and resource interactions," The Engineering Economist, vol. 46, no. 3, pp. 164-180, 2001.

[18] C. Kahraman, D. Ruan, and E. Tolga, "Capital budgeting techniques using discounted fuzzy versus probabilistic cash flows," Information Sciences, vol. 142, no. 1-4, pp. 57-76, 2002.

[19] T.-S. Liou and C.-W. Chen, "Fuzzy decision analysis for alternative selection using a fuzzy annual worth criterion," Engineering Economist, vol. 51, no. 1, pp. 19-34, 2006.

[20] O. A. Omitaomu and A. Badiru, "Fuzzy present value analysis model for evaluating information system projects," Engineering Economist, vol. 52, no. 2, pp. 157-178, 2007.

[21] X. Huang, "Credibility-based chance-constrained integer programming models for capital budgeting with fuzzy parameters," Information Sciences, vol. 176, no. 18, pp. 2698-2712, 2006.

[22] X. Huang, "Change-constrained programming models for capital budgeting with NPV as fuzzy parameters," Journal of Computational and Applied Mathematics, vol. 198, no. 1, pp.149159, 2007.

[23] X. Huang, "Optimal project selection with random fuzzy parameters," International Journal of Production Economics, vol. 106, no. 2, pp. 513-522, 2007.

[24] R. Goetschel Jr. and W. Voxman, "Elementary fuzzy calculus," Fuzzy Sets and Systems, vol. 18, no. 1, pp. 31-43, 1986.

[25] W.-G. Zhang and Z.-K. Nie, "On possibilistic variance of fuzzy numbers," in Proceedings of the 9th International Conference on Rough Sets, Fuzzy Sets, Data Mining, and Granular Computing (RSFDGrC '03), vol. 2639 of Lecture Notes in Artificial Intelligence, pp. 398-402, May 2003.

[26] I. Georgescu, "Possibilistic risk aversion," Fuzzy Sets and Systems, vol. 160, no. 18, pp. 2608-2619, 2009.

[27] S. S. Appadoo, C. R. Bector, and V. N. Sharma, Net Present Value under Fuzzy Data, Administrative Sciences Association of Canada (ASAC), University of Quebec at Montreal (UQUAM), Montreal, Canada, 2000. 


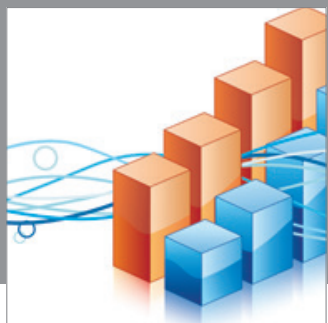

Advances in

Operations Research

mansans

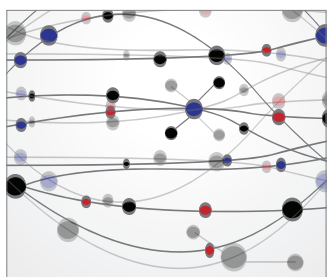

The Scientific World Journal
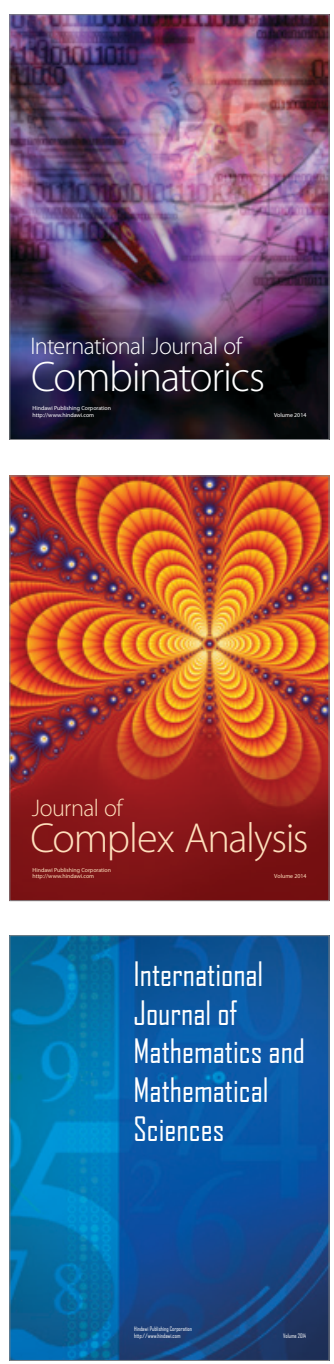
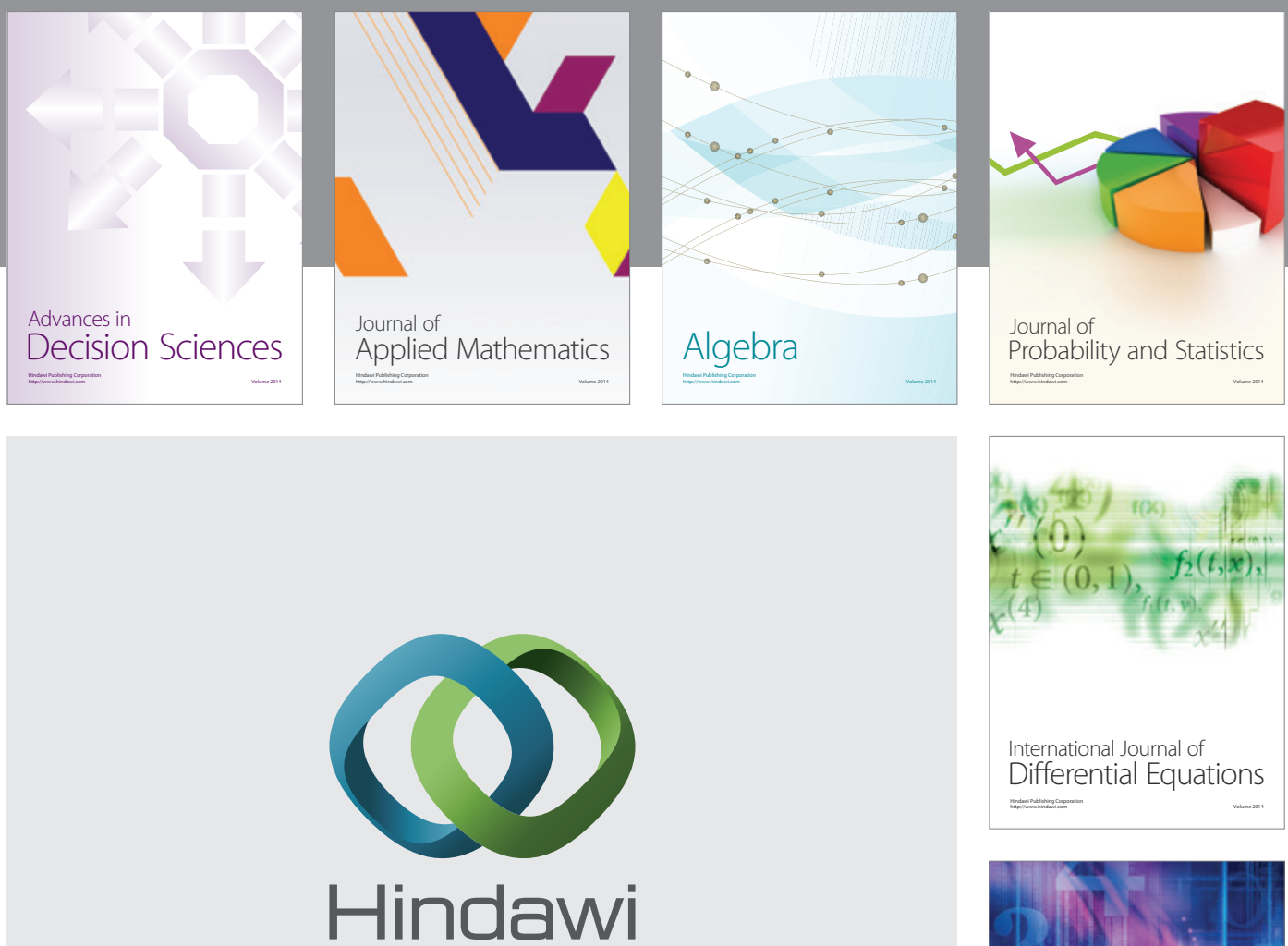

Submit your manuscripts at http://www.hindawi.com
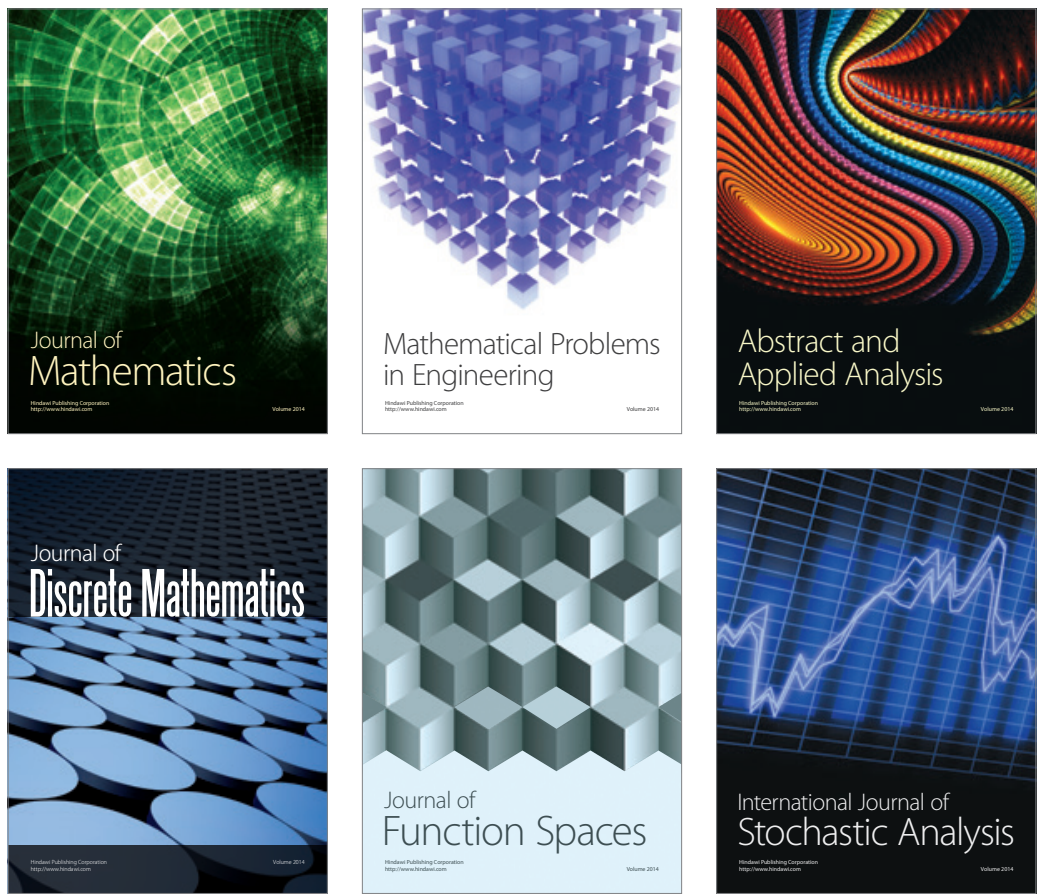

Journal of

Function Spaces

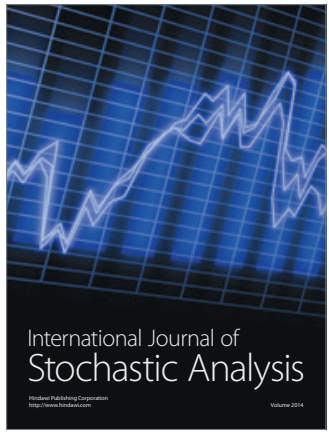

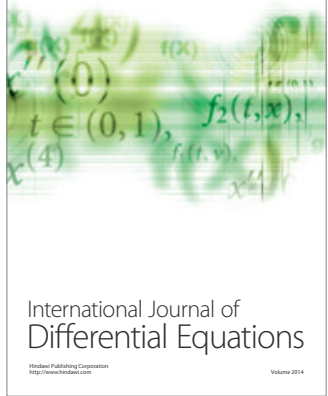
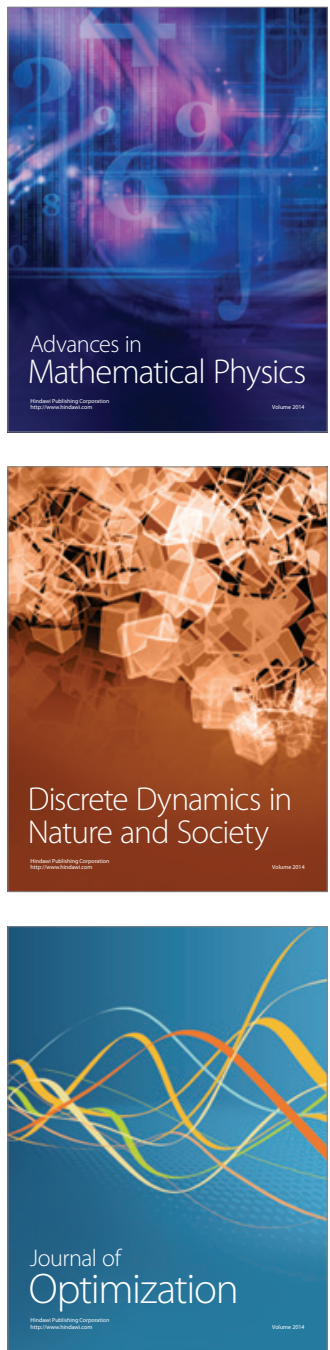